Cancer and Workplace Chemicals. JH Duffus. HHSC Handbook No 17. (Pp 75 +8 tables; £15.00.) 1995. ISBN 0-94823726-0.

This is a small, short book with a spring binding that fits comfortably into a lab coat pocket. It reviews "current thinking relating to the role of workplace chemicals in causing cancer and how it relates to the regulation... (of) carcinogens (in the workplace)".

Its layout is easy to follow and the text is easy to read. Large print ensures that all details relating to numerous lengthy and important directives are readily appreciated. There is a good list of references and a workmanlike, but not exhaustive, glossary of terms relating to carcinogenesis. The tables given are similarly clearly presented and easy to follow. This clarity of style ensures that the book can be used for quick reference. It suffers from not listing the chemicals covered in a glossary or all of them in its index.

Probably because of its small size, the text is quite general when describing mechanisms of carcinogenesis but nevertheless is nicely informative for undergraduates or for just dipping into. The section on classification of carcinogens is worth reading if only to remind oneself of the problems in this area and national differences.

The heart of the book is the coverage of the legislation controlling the use of carcinogens and related substances. Many of these substances are well known but listing their many relevant regulations should be an attraction to health and safety officers and staff likely to handle them. If for no other reason this book should find a ready use for identifying and cross checking what is a complex area of essential regulatory control for occupational risks. Its clarity and good general coverage of an important area in health and safety are also worth considering.

\section{B RUSHTON}

Standard Haematology Practice 2. Ed K Wood. (Pp 289; £49.50.) Blackwell Science Ltd. 1995. ISBN 0-632-03739-3.

The 22 chapters of the book are guidelines on a variety of haematological topics prepared by the Task Force of the British Committee for Standards in Haematology which itself is a subcommittee of the British Society for Haematology. As the membership of the various task forces consists of the leading authorities in Britain in their respective subbranches of haematology, this book can be considered to reflect current thinking and practice in haematology in this country. Trained and trainee haematologists together with medical laboratory scientists should therefore have a copy available to them in order to assess whether they are maintaining adequate standards of work and, if they find they are not, then goals are provided for which they must aim. Every chapter provides a sound basis for good quality audit sessions and the final chapter even informs the reader how to approach the concept of audit and successfully carry it out. Although one might expect guidelines written by Committees to be worthy but dull, most, but not all of the chapters are lively, succinct and easy to read. This especially applies to the 15 guidelines relating to specific topics in general haematology, haemostasis and thrombosis and blood transfusion. Seven chapters deal with subjects relating to haematology laboratory management and discuss the managerial skills which must be acquired by senior medical and technical staff to ensure that the laboratories for which they are responsible provide a safe and efficient haematology service. With the increasing trend for hospital managers to look toward market forces with their emphasis on costs, it is imperative that work of high quality as reflected in these guidelines remains the prime objective of all grades of staff who work in haematology and this book, in conjunction with its companion volume, provides a sound basis for achieving this ideal.

\section{A WINFIELD}

Colour Atlas of Ocular Manifestations of AIDS. Diagnosis and Management. J Orellana, RM Lieberman, SA Teich. (Pp 105; f108.00.) Igaku-Shoin. 1995. ISBN 4-26014273-9.

The eye is often described as a window on the world, but it is also a window on the body and in AIDS allows the ophthalmologist to see many of the processes which characterise the immunodeficiency related disorders of HIV infection. The book starts with a good review section, although most readers would have to be seriously out of date to have missed most of this elsewhere. The chapter on public health concerns is in fact about precautions to prevent transmission by ophthalmic procedures. The ocular manifestations of AIDS are covered by anatomical location rather than by disease or process. This has led to some duplication, but the three authors have divided the problems well between the chapters, so this is not too noticeable. The final chapter on systemic manifestations might be needed by some ophthalmologists whose clinics are commonly held in gloomy conditions guaranteed to dilate the pupils, but not very good for dermatology!

A considerable number of histopathology photographs are included, and most of these are of high quality although in some the colour balance could be improved. Atlas textbooks are usually expensive for their size because of the need for high quality colour reproduction. This book is no exception, but the illustrations are generally very good-better than some pathology atlases!

\section{A CREE}

Current Clinical Topics in Infectious Diseases. 14. Eds JS Remington, MN Swartz. (Pp 296; £59.50.) Blackwell Scientific Publications. 1994. ISBN 0-86542359-8.

This is the most recent in an excellent series of books which have come out more or less annually. The subjects are wide ranging but do reflect current concerns. All the authors are senior and well respected in their fields. The reviews are excellent. Kurchner and Gibbons (Boston) set off with foot infections in diabetics and the advice about early and aggressive, curative surgery is important. The clinical subjects covered also include splenic abscess (a change in predominant pathogens in recent years), human herpesvirus 6 , MRSA, and Rochalimaea spp infections. This genus has now been included in Bartonella. Of the antimicrobial subjects, there are reviews of automation of antibiotic sensitivity testing, new macrolides, cytokine therapy (not much here except for rather specialist applications such as interferon for hepatitides and CSFs for neutropenics), and intelligent dosing with antimicrobials, much of which is about single daily doses of aminoglycosides. Infection control is represented in an article on the prevention of HIV infection in health care workers. Two more general chapters are about new vaccine development and ethical issues. This last chapter deals very briefly with the concepts of medical ethics in general and then presents some case histories suggesting a profound ethical dilemma in management.

I sometimes wonder about the value of books which contain a series of articles about what the editors perceived to be topical or debatable subjects. I use the past tense advisedly - by the time the book comes out, because of the inevitable delays in publication, things are likely to be rather out of date. Before you rush out to buy this volume there will have been additional delay (down to your reviewer) getting this review into print.

The obvious value is that each chapter gives an overview of subject. The author may be an expert or may be someone whom the editors managed to persuade to write a chapter. We could audit the book according to recency of references or by the number of papers written by the chapter author(s) (Should the author quote his own work or be modest and review the work of others?) or search out unreferenced work which is the author's unattributed recent contribution to the genre.

If you get asked to write a chapter, remember that it will get you $10 \mathrm{CME}$ points but a book will only earn 50 points-best to write more than five chapters and get points for each.)

What of this book? It is the 14th in a prestigious series of infection books. It is unashamedly American in style and content and there are some differences in the ways that we approach infectious diseases. A review of the chapter on medical ethics is very in teresting in this respect. It is a good bedside read and if you are setting off to research one of the topics, each chapter provides a reasonable list of references (but virtually none more recently than 1992) which will act as a respectable starting point for a literature search.

\section{G M SCOTT}

Diagnostic Bacteriology Protocols. Methods in Molecular Biology. Vol 36. Eds Jenny Howard, David $M$ Whitcombe. (Pp 283; soft cover - no price given.) Humana Press. 1995. ISBN 0896032973.

Diagnostic Bacteriology Protocols is the 36th in the series of Methods in Molecular Biology. The authors set themselves an ambitious aim presenting a broad range of techniques for detecting, identifying and differentiating bacterial cell components. The protocols are intended to be easy to follow and "guarantee reproducible results for novices and senior researchers alike". If only that were true! Prospective purchasers should not be discouraged by this hype. In many respects the authors provide protocols which are clear to follow and should prove useful to a wide range of researchers.

The title is somewhat misleading as it may appear this book is suitable for those planning 